\title{
On Separation between Metric Observers in Segal's Compact Cosmos
}

\author{
Alexander Levichev ${ }^{1}$, Andrey Palyanov ${ }^{2}$ \\ ${ }^{1}$ Sobolev Institute of Mathematics SB RAS, Novosibirsk, Russia \\ ${ }^{2}$ A. P. Ershov Institute of Informatics Systems SB RAS, Novosibirsk, Russia \\ Email: alevichev@gmail.com
}

Received 14 July 2015; accepted 14 November 2015; published 17 November 2015

Copyright (C) 2015 by authors and Scientific Research Publishing Inc.

This work is licensed under the Creative Commons Attribution International License (CC BY).

http://creativecommons.org/licenses/by/4.0/

(c) (i) Open Access

\section{Abstract}

A certain class $\mathrm{K}$ of GR homogeneous spacetimes is considered. For each pair $E, \tilde{E}$ of spacetimes from $\mathrm{K}, \tilde{E}=g(E)$ where conformal transformation $g$ is from $\mathrm{G}=S U(2,2)$. Each $E$ (being $U(2)$ or its double cover, as a manifold) is interpreted as related to an observer in Segal's universal cosmos. The definition of separation $d$ between $E$ and $\tilde{E}$ is based on the integration of the conformal factor of the transformation $g$. The integration is carried out separately over each region where the conformal factor is no less than 1 (or no greater than 1). Certain properties of $d=d(E, \tilde{E})$ are proven; examples are considered; and possible directions of further research are indicated.

\section{Keywords}

Separation between Spacetimes, Segal's Universal Cosmos, Conformal Group Action on U(2), DLF-Theory

\section{Motivation and Introduction}

The first author has been interested in GR ("GR" is for General Relativity) research for quite a while and he concentrated on a few most symmetric spacetimes ([1], [2], and more). Later (see [3], [4]) he has become a strong believer in Segal's Chronometric Theory (see [5], electronic archive arranged by Levichev), and he is attempting to modify Segal's Theory (see [6], a key publication). The collaboration of the two current authors is based on their mutual interest in Penrose-Hameroff approach to consciousness (see its update in [7], [8]). Specifically, we are putting forward an alternative definition of separation between space-times. In [9], the original definition was based on bringing up a Newtonian limit in GR. Our definition has been introduced in 
[10], [11], and we now present it in much more detail.

Recall the Lie group $U(2)$ as the totality of all two-by-two matrices $z$ (with complex entries allowed) satisfying

$$
Z^{*} Z=1 \text {, }
$$

where $z^{*}$ is the transpose and complex conjugate of $z$, and $\mathbf{1}$ is the unit matrix. Now, define the Lie group $\mathrm{G}=S U(2,2)$ as consisting of all four-by-four matrices $g$ (with complex entries allowed) satisfying

$$
g^{*} S g=S,
$$

where $S$ is the diagonal matrix $\operatorname{diag}\{1,1,-1,-1\}$. Recall the well-known linear-fractional $G$-action on $U(2)$ :

$$
g(z)=(A z+B)(C z+D)^{-1}
$$

where a matrix $g$ from $G$ is determined by its $2 \times 2$ blocks $A, B, C, D$.

In Table I of [12], the matrices $\boldsymbol{L}_{i j}$ are chosen as basic vectors of the (fifteen-dimensional) Lie algebra $s u(2,2)$, whereas $L_{i j}$ are the corresponding vector fields on $U(2)$. The vector fields $L_{i j}$ are determined by the $G$-action (1.3). As explained in [12], subscripts $i, j$ take on $-1,0,1,2,3,4$, and the convention $\boldsymbol{L}_{j i}=-\boldsymbol{L}_{i j}$ (resulting in $L_{j i}=-L_{i j}$ ) holds.

The Lorentzian inner product on $U(2)$ is introduced in such a way that left-invariant vector fields $X_{0}=L_{-10}, X_{1}=L_{14}-L_{23}, X_{2}=L_{24}-L_{31}, X_{3}=L_{34}-L_{12}$ form an orthonormal basis (following [12], we use +, - , -, - signature). The resulting product on $U(2)$ is bi-invariant (see [6]), and $\langle\mathbf{a}, \mathbf{b}\rangle$ below denotes the Lorentzian inner product of tangent vectors $\mathbf{a}, \mathbf{b}$ at a point $z$ of $U(2)$. The spacetime thus obtained is denoted by $E_{0}$ (the meaning of the subscript will become clear in the next section). Transformations (1.3) are conformal in $E_{0}$ (a word of caution: this spacetime has been denoted as $D$ in [6]). As it follows from Table I of [12], the vector fields $L_{-10}, L_{12}, L_{13}, L_{14}, L_{23}, L_{24}, L_{34}$, generate isometries in $E_{0}$. The corresponding subgroup $\mathrm{K}$ in $G$ consists of all matrices (1.2) with $B=C=0$.

For what follows, it is instrumental to introduce a certain bi-invariant Riemannian inner product on $U(2)$. To do so, we recall that vector fields $X_{0}, X_{1}, X_{2}, X_{3}$ constitute a basis of the Lie algebra $u(2)$. This algebra is a direct sum of its center with $s u(2)$. Namely, $X_{0}$ generates the center, whereas $X_{1}, X_{2}, X_{3}$ are basic vectors in $s u(2)$. The Riemannian metric is determined by the demand on left-invariant vector fields $X_{0}, X_{1}$, $X_{2}, X_{3}$ to form an orthonormal basis. The corresponding Riemannian space is denoted $E_{R}$. Again, $E_{R}$ is $U(2)$, as a manifold. Our (a,b) below denotes the Riemannian inner product of tangent vectors $\mathbf{a}, \mathbf{b}$ at a point $z$ of $U(2)$. In the forthcoming sections the corresponding volume form on $E_{R}$ will be instrumental. From Table I of [12], it follows that the group $\mathrm{K}$ acts as a group of both Lorentzian and Riemannian isometries.

Notice that, as a group, $U(2)$ is not a direct product of its center with the subgroup $S U(2)$. The double cover $E^{(2)}$ of $U(2)$ is the direct product $S^{1} \times S^{3}$ (with $S^{3}$ represented by $S U(2)$ ). The covering map sends $\left(e^{i t}, u\right)$ into the matrix $e^{i t} u$ in $U(2)$. The corresponding Lorentzian metric on $E^{(2)}$ is of the form

$$
(d t)^{2}-(d u)^{2} \text {. }
$$

Here the variable $t$ is along $S^{1}$ whereas $(d u)^{2}$ is for the standard Riemannian metric on $S^{3}$. More details are given in our Appendix A, where $u$ denotes a matrix from $S U(2)$. Our Appendix B is dedicated to a certain one-parameter group of transformations (1.3).

It is well-known ([12], [13]) that the (above introduced) covering map is a Lorentzian isometry. Infinitesimal $G$-action on $E^{(2)}$ is presented in Table I of [12]. It is known (see [13]) that action (1.3) can be lifted to a global conformal $G$-action on $E^{(2)}$. Using the corresponding commutative diagram, one can show that the lifted action of the group $\mathrm{K}$ is as follows:

$$
\left(e^{i t}, u\right) \rightarrow\left((\operatorname{det} A) e^{i t},(\operatorname{det} A)^{-1} A u D^{-1}\right) .
$$

Also, it is easily verifiable that for the Riemannian metric

$$
(d t)^{2}+(d u)^{2}
$$

on $S^{1} \times S^{3}$ the (above specified) covering map is a Riemannian isometry from $E^{(2)}$ onto $E_{R}$.

It makes sense to mention how a suitable version of the Einstein static universe, $E_{u c}$, can be introduced in the 
context of our work (the subscript $u c$ is for universal cover). To be more precise, $E_{u c}$ should be called universal cosmos ([14]) or Segal's universal cosmos ([13]). The universal cover $E_{u c}$ of $E_{0}$ is $R^{1} \times S^{3}$, topologically. The $G$-action on $E_{0}$ is canonically lifted to the $G_{u c}$-action on $E_{u c}$ (the latter action preserves the causal structure of $E_{u c}$ ). In a cosmological model based on $E_{u c}$, there is a conformal invariant $R$, interpreted as the radius of a three-dimensional (physical) space $S^{3}$. I. Segal (in [14] and in other publications) has put this $R$ for the (long wanted by Dirac and others) third fundamental constant additionally to the speed of light and to the Planck's constant. It is known that to model particles on $E_{u c}$, one can start with the world $E_{0}$, a compact one. The respective property is called automatic periodicity ([15], p. 202), and it allows us to only deal with compact spacetimes $E_{0}$ and $E^{(2)}$ (which explains our compact Segal's cosmos terminology).

More precisely, we deal with two classes of spacetimes: $\mathbf{K}_{1}$ and $\mathbf{K}_{2}$. Roughly speaking, the first class is obtained by application of all transformations (3) to $E_{0}$; details follow in the next section (where $\mathbf{K}_{2}$ will also be defined).

\section{On the Notion of Separation between Spacetimes: The Main Definition and Related Properties}

The separation (or distance) $d(x, y)$ will be defined for any pair $x, y$ of spacetimes from $\mathbf{K}_{1}$ (or from $\mathbf{K}_{2}$ ).

As mentioned, the totality of all isometries in $E_{0}$ is the group $\mathrm{K}$ of all matrices (1.2) with $B=C=0$. Each member $E$ of the class $\mathbf{K}_{1}$ will be now put in correspondence with an element $x$ of the homogeneous space $\mathrm{G} / \mathrm{K}$. Namely, each element (or coset) $x$ of $\mathrm{G} / \mathrm{K}$ is specified by an element $g$ from G: $x=g \mathrm{~K}=\{g k: k \in \mathrm{K}\}$. One and the same $x$ can be determined by another element (say, $\left.g_{1}\right)$ from G: $g \mathrm{~K}=g_{1} \mathrm{~K}$. For such a pair $\left(g, g_{1}\right)$, there exists such $k$ from $K$, that $g_{1}=g k$. When the subgroup $K$ is viewed as an element of $G / K$, denote $K$ as $x_{0}$. This $x_{0}$ we put into correspondence with $E_{0}$ (which has been described in Section 1). As a manifold, each element $x$ of $\mathbf{K}_{1}$ is $U(2)$. In what follows, we use $\langle\mathbf{a}, \mathbf{b}\rangle$ (rather than $\langle\mathbf{a}, \mathbf{b}\rangle_{0}$ ) to denote the (Lorentzian) inner product of vectors $\mathbf{a}, \mathbf{b}$ from the tangent space $T\left(E_{0}\right)$ at $z$. This inner product has been introduced in our Section 1. To define spacetime $E$ corresponding to a coset $x=g \mathrm{~K}$, it is enough to specify the inner product $\langle. . .\rangle_{E}$, see (2.2) below. Such a transformation $g$ is conformal in $E_{0}$. Namely, given vectors a,b from the tangent space $T\left(E_{0}\right)$ at $z$, the inner product $\left\langle g_{*} \mathbf{a}, g_{*} \mathbf{b}\right\rangle$ at $g(z)$ of their images (under the tangent map $g_{*}$ ) satisfies

$$
\left\langle g_{*} \mathbf{a}, g_{*} \mathbf{b}\right\rangle=h(z)\langle\mathbf{a}, \mathbf{b}\rangle .
$$

The everywhere positive function $h(z)$ is known as the square of conformal coefficient. Frequently, we will simply refer to this $h(z)$ as to a conformal coefficient. Given vectors a,b from the tangent space $T(E)$ at $z$, their inner product can be defined as follows:

$$
\langle\mathbf{a}, \mathbf{b}\rangle_{E}=\left[h\left(g^{-1}(z)\right)\right]^{-1}\langle\mathbf{a}, \mathbf{b}\rangle,
$$

where $\langle\mathbf{a}, \mathbf{b}\rangle$ is calculated at $z$. Notice, that $h$ here is the (above mentioned) function on $U(2)$ determined by $g$ It is easy to show that (2.2) is equivalent to the condition for $g$ to be an isometry between $\left(E_{0},\langle.,\rangle.\right)$ and $\left(E,\langle., .\rangle_{E}\right)$ :

$$
\left\langle g_{*} \mathbf{a}, g_{*} \mathbf{b}\right\rangle_{E}=\langle\mathbf{a}, \mathbf{b}\rangle .
$$

Here the right hand side of (2.3L) is calculated in $E_{0}$ at $z$, and it defines the inner product $\left\langle g_{*} \mathbf{a}, g_{*} \mathbf{b}\right\rangle_{E}$ of vectors $g_{*} \mathbf{a}$ and $g_{*} \mathbf{b}$ at $g(z)$. To avoid verification of (2.2)-(2.3L) equivalence, we define the Lorentzian metric in $E$ in terms of (2.3L). Similarly, we define the following Riemannian metric in $E$ :

$$
\left(g_{*} \mathbf{a}, g_{*} \mathbf{b}\right)_{E}=(\mathbf{a}, \mathbf{b}) .
$$

where the positive definite inner product in the right hand side of (2.3R) has been introduced in our Section 1.

Let us show that, given a coset $x$ in $\mathrm{G} / \mathrm{K},(2.3 \mathrm{~L})$ correctly defines a Lorentzian metric on $U(2)$, whereas (2.3R) correctly defines a Riemannian metric on $U(2)$ : 
Scholium 2.1. The inner product (2.3L) (respectively, the inner product (2.3R)) is independent of the choice of $g$ which represents a coset $x$.

Proof. If $x$ is represented as $g_{1} \mathrm{~K}$, then $g_{1}=g k$ where $k$ is a certain element of the group K. Given such a representation, the analogue of $(2.3 \mathrm{R})$ is

$$
\left\{g_{1 * \mathbf{a}}, g_{1^{*}} \mathbf{b}\right\}_{E}=\langle\mathbf{a}, \mathbf{b}\rangle
$$

where the right hand side of (2.4) is calculated in $E_{0}$ at $z$, and it defines the inner product $\left\{g_{1^{*}} \mathbf{a}, g_{1^{*}} \mathbf{b}\right\}_{E}$ of vectors $g_{1^{*}} \mathbf{a}$ and $g_{1^{*}} \mathbf{b}$ at $g_{1}(z)$. We have to show that (2.4) introduces the same metric structure on $U(2)$ as $(2.3 \mathrm{~L})$ does. To do so, we rewrite $(2.3 \mathrm{~L})$ in the form of

$$
\left\{(g k)_{*} \mathbf{a},(g k)_{*} \mathbf{b}\right\}_{E}=\left\langle k_{*} \mathbf{a}, k_{*} \mathbf{b}\right\rangle
$$

where the right hand side is calculated at $k(z)$, and the left hand side is calculated at $g(k(z))$.

However, $\left\langle k_{*} \mathbf{a}, k_{*} \mathbf{b}\right\rangle$ in (2.5) equals $\langle\mathbf{a}, \mathbf{b}\rangle$ in (2.4) since $k$ is a Lorentzian isometry in $E_{0}$. Comparison of (2.4) with (2.5) finishes the proof. The verification process, that (2.3R) is independent of representative, copies the one for $(2.3 \mathrm{~L})$.

Let us notice (see [16]) that each $\left(E,\langle. . .\rangle_{E}\right)$ can be interpreted as a spacetime corresponding to a certain (global) observer. A word of caution: [16] treats the universal cover of $E_{0}$ whereas we only deal with compact spacetimes here.

Remark 2.2. We have thus defined the class $\mathbf{K}_{1}$ of spacetimes. Our class $\mathbf{K}_{2}$ can be similarly introduced in terms of the Lorentzian manifold $E^{(2)}$ (the 2-cover of $E_{0}$ ) with the (lifted) $G$-action on $E^{(2)}$.

Given a (1.3)-transformation $g$ of (Lorentzian) $E_{0}$, define the following subsets of $E_{0}$ :

$$
\begin{aligned}
& T_{g}^{+}=\left\{z: h_{g}(z) \geq 1\right\}, \\
& T_{g}^{-}=\left\{z: h_{g}(z) \leq 1\right\} ;
\end{aligned}
$$

here $h_{g}(z)$ is the square of the conformal coefficient at $z$ of the transformation $g$. A (non-negative) number $d_{g}$ is defined as follows:

$$
d_{g}=\ln \left\{\left[\int h_{g}^{2}\right] /\left[V\left(T_{g}^{+}\right)\right]\right\}+\ln \left\{\left[\int h_{g}^{-2}\right] /\left[V\left(T_{g}^{-}\right)\right]\right\},
$$

where $V(S)$ is for the volume of a set $S$ in $E_{R}$ (with the volume form introduced in our Section 1). Clearly, expressions inside the logarithms in (2.8) can be interpreted as corresponding cumulative distortions of the original metric structure in $E_{0}$. To be sure of convergence of all of the integrals involved, it is enough to mention that each of the two integrands is a continuous function over the corresponding region of integration, whereas each of the regions (2.6), (2.7) is a compact set.

To further deal with (2.8), we now proceed with more technicalities. Clearly, $d_{g}$ can be viewed as $\ln \{(a c) /(b d)\}$ with $b=V\left(T_{g}^{+}\right), d=V\left(T_{g}^{-}\right)$. The integration in $a$ is over $T_{g}^{+}$, whereas in $c$ the integration is over $T_{g}^{-}$.

Examples of integrals $a, b, c, d$ evaluations are given in our Appendix C (see Theorem C.5). Notice that

$$
d_{g}=0 \text { if and only if } g \text { is an isometry of } E_{0} \text {, }
$$

which follows from (2.8) because in this case $h_{g}(z)=1$, a constant function on $U(2)$. As a result of $h_{g}(z)=1$, each of the two terms in the sum (2.8) is zero.

Scholium 2.3. Given the (1.3)-transformation $g$ and isometries $k_{1}, k_{2}$, the following holds:

$$
d_{w}=d_{g},
$$

where $w=k_{1}^{-1} g k_{2}$.

Proof. To prove (2.10), we will now show that each of the four numbers ( $a, b, c$, and $d$ ) remain the same when we switch from $d_{g}$ to $d_{w}$. Namely: 


$$
\begin{aligned}
T_{w}^{+} & =\left\{z: h_{w}(z) \geq 1\right\}=k_{2}^{-1}\left(\left\{k_{2}(z): h_{m}\left(k_{2}(z)\right) \geq 1\right\}\right) \\
& =k_{2}^{-1}\left(\left\{k_{2}(z): h_{g}\left(k_{2}(z)\right) \geq 1\right\}\right), \text { where } m=k_{1}^{-1} g \text {; hence } T_{w}^{+}=k_{2}^{-1}\left(T_{g}^{+}\right) .
\end{aligned}
$$

Similarly, $T_{w}^{-}=k_{2}^{-1}\left(T_{g}^{-}\right)$. Hence, $b=V\left(T_{g}^{+}\right)=V\left(T_{w}^{+}\right)$, and $d=V\left(T_{g}^{-}\right)=V\left(T_{w}^{-}\right)$, due to the K-invariance of the volume form.

Let us now use the variable $k_{2}(z)$ in the integral $a$ of $\left(h_{w}\right)^{2}$ over $T_{w}^{+}$: the integrand is then $\left(h_{m}\left(k_{2}(z)\right)\right)^{2}=\left(h_{g}\left(k_{2}(z)\right)\right)^{2}$, the region of integration is $k_{2}\left(T_{w}^{+}\right)=T_{g}^{+}$, and there is no extra factor in the integrand since $k_{2}$ is a transformation from the group K. Similarly, number $c$ remains the same when we switch from $d_{g}$ to $d_{w}$.

Now, if two cosets are represented as $x=g_{1} \mathrm{~K}, y=g_{2} \mathrm{~K}$, define the distance $d(x, y)$ as

$$
d(x, y)=d_{g} \text {, }
$$

where $g=\left(g_{1}\right)^{-1} g_{2}$. The number $d(x, y)$ is independent of representatives since if $x$ is represented by $g_{1} k_{1}$, and $y$ is represented by $g_{2} k_{2}$, then for $w=\left(g_{1} k_{1}\right)^{-1} g_{2} k_{2}=k_{1}^{-1} g k_{2}, d_{w}=d_{g}$ according to (2.10).

Corollary 2.4. In the above settings, $d(x, y)=d\left(x_{0}, q\right)$ where $x_{0}=\mathrm{K}$ and $q=g_{1}^{-1} g_{2} \mathrm{~K}$.

A word of caution: we use the term distance but we are not sure that the corresponding triangle inequality holds (even locally) for (2.11). However, we prove (below) that (2.11) is symmetric: $d(x, y)=d(y, x)$, and G-invariant:

$$
d(f(x), f(y))=d(x, y),
$$

for arbitrary $f$ from $G$ (where we have in mind the canonical action of $G$ in $G / K$ ).

As regards $G$-invariance, one can think of a possible relation of our definition (2.11) to the canonical inner product in the symmetric space $G / K$. This we do not discuss here.

Scholium 2.5. The distance (2.11) is symmetric: $d(x, y)=d(y, x)$.

Proof. As justified by our Corollary 2.4, assume that $x=\mathrm{K}$ and $y=g \mathrm{~K}$. Define

$$
T_{m}^{+}=\left\{\tilde{z}: h_{m}(\tilde{z}) \geq 1\right\}, T_{m}^{-}=\left\{\tilde{z}: h_{m}(\tilde{z}) \leq 1\right\},
$$

where $m=g^{-1}$, and where we use $\tilde{z}$ (rather than $z$, as before) to denote a matrix in $U(2)$. Tilde (below) indicates that computations are performed in $E$ rather than in $E_{0}$.

For $d_{m}=\ln \left\{\left[\int\left(h_{m}\right)^{2}\right] /\left[\tilde{V}\left(T_{m}^{+}\right)\right]\right\}+\ln \left\{\left[\int\left(h_{m}\right)^{-2}\right] /\left[\tilde{V}\left(T_{m}^{-}\right)\right]\right\}$, the following is true:

$\tilde{b}=\tilde{V}\left(T_{m}^{+}\right)=\tilde{V}\left(g\left(T_{g}^{-}\right)\right)=V\left(T_{g}^{-}\right)=d$ since, due to (2.3R), $g$ is an isometry between the two Riemannian spaces. Similarly, $\tilde{d}=\tilde{V}\left(T_{m}^{-}\right)=\tilde{V}\left(g\left(T_{g}^{+}\right)\right)=V\left(T_{g}^{+}\right)=b$. The new integrand is then $\left(h_{g}(z)\right)^{-2}$, the new region of integration is $T_{g}^{-}$, and there is no extra factor in the integrand since $g$ is an isometry between the two Riemannian spaces in question. We have thus proven that $\tilde{a}=c$. Similarly, $\tilde{c}=a$. We have thus proven the equality $(\tilde{a} / \tilde{b})(\tilde{c} / \tilde{d})=(a / b)(c / d)$, which results in $d(x, y)=d(y, x)$, the symmetry property of the distance between spacetimes.

\section{Concluding Remarks and Future Research Insights}

Examples of integrals $a, b, c, d$ evaluations (in case of a certain one-parameter group of conformal transformations) are given in our Appendix C. It is of interest to know whether Theorem C.5 holds for other transformations from $G=S U(2,2)$. Evaluations in Appendix C indicate that definition (2.11) of distance between spacetimes seems to be quite a working one. As part of future research, it will be of interest to apply our definition in the case where the original spacetime is $F$ (here we refer to the DLF-theory, [6]). In that case, the underlying manifold is (non-compact!) $U(1,1)$, rather than $U(2)$. Preliminary calculations indicate that a 
conformal coefficient might be unbounded. We will thus have to deal with improper 4D integrals, and the question of convergence will have to be studied first.

\section{References}

[1] Guts, A.K. and Levichev, A.V. (1984) On the Foundations of Relativity Theory. Doklady Akademii Nauk SSSR, 277, 253-257. (In Russian)

[2] Levichev, A.V. (1989) On the Causal Structure of Homogeneous Lorentzian Manifolds. General Relativity \& Gravity, 21, 1027-1045. http://dx.doi.org/10.1007/BF00774087

[3] Levichev, A.V. (1993) The Chronometric Theory by I. Segal Is the Crowning Accomplishment of Special Relativity. Izvestiya Vysshikh Uchebnykh Zavedenii Fizika, 8, 84-89. (In Russian)

[4] Levichev, A.V. (1995) Mathematical Foundations and Physical Applications of Chronometry. In: Hilgert, J., Hofmann, K. and Lawson, J., Eds., Semigroups in Algebra, Geometry, and Analysis, de Gryuter Expositions in Mathematics, Berlin, 77-103. http://dx.doi.org/10.1515/9783110885583.77

[5] http://dedekind.mit.edu/segal-archive/index.php

[6] Levichev, A.V. (2011) Pseudo-Hermitian Realization of the Minkowski World through DLF Theory. Physica Scripta, 83, 1-9. http://dx.doi.org/10.1088/0031-8949/83/01/015101

[7] Hameroff, S. and Penrose, R. (2014) Consciousness in the Universe: A Review of the "Orch OR” Theory. Physics of Life Reviews, 11, 39-78. http://dx.doi.org/10.1016/j.plrev.2013.08.002

[8] Hameroff, S. and Penrose, R. (2014) Reply to Criticism of the “Orch OR Qubit”- “Orchestrated Objective Reduction” Is Scientifically Justified. Physics of Life Reviews, 11, 94-100. http://dx.doi.org/10.1016/j.plrev.2013.11.013

[9] Penrose, R. (1992) Gravity and Quantum Mechanics. In: Gleiser, R.J., Kozameh, C.N. and Moreschi, O.M., Eds., General Relativity and Gravitation 13. Part 1: Plenary Lectures 1992. Proceedings of the 13th International Conference on General Relativity and Gravitation, Cordoba, 28 June-4 July 1992, 179-189.

[10] Levichev, A. and Palyanov, A. (2014) On a Modification of the Theoretical Basis of the Penrose-Hameroff Model of Consciousness. In: International Conference MM-HPC-BBB-2014, Abstracts, Sobolev Institute of Mathematics SB RAS, Institute of Cytology and Genetics, Siberian Branch of the Russian Academy of Sciences, Novosibirsk, 49.

[11] Levichev, V. and Palyanov, A.Yu. (2014) On a Notion of Separation between Space-Times. In: Geometry Days in Novosibirsk. Abstracts of the International Conference, Sobolev Institute of Mathematics, Siberian Branch of the Russian Academy of Sciences, Novosibirsk, 110.

[12] Segal, I.E., Hans, P.J., Bent, Ø., Paneitz, S.M. and Speh, B. (1981) Covariant Chronogeometry and Extreme Distances: Elementary Particles. PNAS, 78, 5261-5265. http://dx.doi.org/10.1073/pnas.78.9.5261

[13] Werth, J.-E. (1986) Conformal Group Actions and Segal’s Cosmology. Reports on Mathematical Physics, 23, $257-268$. http://dx.doi.org/10.1016/0034-4877(86)90023-6

[14] Segal, I.E. (1976) Mathematical Cosmology and Extragalactic Astronomy. Academic Press, New York.

[15] Branson, T.P. (1987) Group Representations Arising from Lorentz Conformal Geometry. Journal of Functional Analysis, 74, 199-291. http://dx.doi.org/10.1016/0022-1236(87)90025-5

[16] Segal, I.E. (1984) Evolution of the Inertial Frame of the Universe. Nuovo Cimento, 79B, 187-191. http://dx.doi.org/10.1007/BF02748970

[17] Kon, M. and Levichev, A. (2015) Towards Analysis in Space-Time Bundles Based on Pseudo-Hermitian Realization of the Minkowski Space. In Preparation. 


\section{Appendix A: Parameterizations of $U(2)$ and $E^{(2)}$}

The following presentation for $E^{(2)}$, the 2-cover of $U(2)$, has been widely used in the literature. Consider the direct sum $E^{6}=E^{2} \oplus E^{4}$ of two Euclidean spaces: $E^{2}$ with rectangular coordinates $u_{-1}$, $u_{0}$, and $E^{4}$ with rectangular coordinates $u_{1}, u_{2}, u_{3}, u_{4}$. Each "event" in $E^{(2)}$ is a 6-tuple $\left(u_{-1}, u_{0}, u_{1}, u_{2}, u_{3}, u_{4}\right)$, satisfying

$$
u_{-1}^{2}+u_{0}^{2}=1
$$

and

$$
u_{1}^{2}+u_{2}^{2}+u_{3}^{2}+u_{4}^{2}=1 .
$$

Clearly, $E^{(2)}$ is $S^{1} \times S^{3}$, topologically. The earlier introduced $e^{i t}$ (see Section 2) is $u_{-1}+i u_{0}$, whereas the matrix $u$ from $S U(2)$ is specified as follows:

$$
u=\left[\begin{array}{cc}
u_{4}+i u_{3} & u_{2}+i u_{1} \\
i u_{1}-u_{2} & u_{4}-i u_{3}
\end{array}\right]
$$

The covering map from $\mathrm{E}^{(2)}$ onto $U(2)$ takes the pair $\left(u_{-1}+i u_{0}, u\right)$ into the matrix $\left(u_{-1}+i u_{0}\right) u$, an element $z$ of the group $U(2)$ :

$$
z=\left(u_{-1}+i u_{0}\right) u
$$

Given a matrix $z$ in $U(2)$, the factors $\left(u_{-1}+i u_{0}\right)$ and $u$ are defined up to a sign, only. In terms of $E^{6}$, it is helpful to consider a pseudo-Euclidean metric

$$
\left(d u_{-1}\right)^{2}+\left(d u_{0}\right)^{2}-\left(d u_{1}\right)^{2}-\left(d u_{2}\right)^{2}-\left(d u_{3}\right)^{2}-\left(d u_{4}\right)^{2}
$$

and an Euclidean metric

$$
\left(d u_{-1}\right)^{2}+\left(d u_{0}\right)^{2}+\left(d u_{1}\right)^{2}+\left(d u_{2}\right)^{2}+\left(d u_{3}\right)^{2}+\left(d u_{4}\right)^{2} .
$$

It is known (see [14], p. 40) that the restriction of (A5L) onto $E^{(2)}=S^{1} \times S^{3}$ coincides with metric (1.4) of our Section 1. Similarly, the restriction of (A5R) onto $E^{(2)}$ coincides with metric (1.5).

\section{Appendix B: The Case of a Certain One-Parameter Group of Conformal Transformations}

This group consists of all (1.3)-transformations $g$ of the form:

$$
g=\left[\begin{array}{ll}
A & B \\
C & D
\end{array}\right]
$$

with

$$
A=\left[\begin{array}{ll}
1 & 0 \\
0 & c
\end{array}\right], B=\left[\begin{array}{ll}
0 & 0 \\
s & 0
\end{array}\right], C=\left[\begin{array}{ll}
0 & s \\
0 & 0
\end{array}\right], D=\left[\begin{array}{ll}
c & 0 \\
0 & 1
\end{array}\right],
$$

where $c=\cosh \tau, \quad s=\sinh \tau, \tau$ being a real parameter. This subgroup is contained in a (two-dimensional) subgroup A (from the Iwasawa decomposition $S U(2,2)=\operatorname{KAN}$ ). The $f(z)$ below is for the (positive) square root of the conformal factor $h(z)$. The latter has been defined by our (2.1). To simplify notation, we, sometimes, use the same symbol (like $z$ or $\tilde{z}$, below) to denote both an element of $E^{(2)}$ and a matrix in $E_{0}$. The statement and the proof of the following theorem presume usage of rectangular coordinates in Euclidean $E^{6}$ : see Appendix A.

Theorem B.1. The image $\tilde{z}$ of $z$ in $E^{(2)}$ and the conformal factor $h(z)$ at $z$ (under the lift of the (B.1)transformation $g$ ) are as follows:

$$
\left.\begin{array}{l}
\tilde{u}_{-1}+i \tilde{u}_{0}=\left[c u_{-1}-s u+i\left(c u_{0}-s u_{1}\right)\right] f(z), \\
\tilde{u}_{2}+i \tilde{u}_{1}=\left[c u_{2}-s u_{-1}+i\left(c u_{1}-s u_{0}\right)\right] f(z), \\
\tilde{u}_{4}+i \tilde{u}_{3}=\left(u_{4}+i u_{3}\right) f(z)
\end{array}\right\}
$$




$$
h(z)=(f(z))^{2}=\left[\left(c u_{-1}-s u_{2}\right)^{2}+\left(c u_{0}-s u_{1}\right)^{2}\right]^{-1} .
$$

Proof. Notice that due to (A3) and (A4) from Appendix A, the formulas (B.2) correctly define the transformation on the level of $E_{0}$ (when $z$ and $\tilde{z}$ are matrices). To prove this first part, we use (B.1) in a straightforward way and (omitting routine details of the calculation) determine (B.2). At this stage of the proof we cannot be sure that $h(z)$ is the conformal coefficient. To prove that it is, apply the differential operator $d$ to both sides of (B.2) in order to express

$$
\left(d \tilde{u}_{-1}\right)^{2}+\left(d \tilde{u}_{0}\right)^{2}+\left(d \tilde{u}_{1}\right)^{2}-\left(d \tilde{u}_{2}\right)^{2}-\left(d \tilde{u}_{3}\right)^{2}-\left(d \tilde{u}_{4}\right)^{2}
$$

in terms of differentials $d u_{-1}, d u_{0}, d u_{1}, d u_{2}, d u_{3}, d u_{4}$. Comparison of the obtained expression with (A5L) verifies (B.3).

Remark B.2. In the case considered, there is an alternative way to determine the conformal factor (B.3). It is as follows [17], Theorem 3: for a (1.3)-transformation $g$, the following equality holds for the conformal factor at $z$ :

$$
h(z)=\operatorname{det}\left(\left[A-(A z+B)(C z+D)^{-1} C\right] z(A z+B)^{-1}\right) .
$$

One can verify that (B.5), when applied in the (B.1)-case, results in (B.3).

It is of interest to determine all fixed points (that is, matrices $z=\left[\begin{array}{ll}z_{1} & z_{2} \\ z_{3} & z_{4}\end{array}\right]$ with $g(z)=z$ property) of the transformation (B.1).

Scholium B.3. The totality of all fixed points of (B.1) is a pair of circles. One of the circles is given by equations $z_{3}=1, z_{1}=z_{4}=0$. The other circle is given by equations $z_{3}=-1, z_{1}=z_{4}=0$.

Proof. As it follows from (B.1), the totality of all fixed points is the solution set of

$$
\left[\begin{array}{ll}
z_{1} & z_{2} \\
z_{3} & z_{4}
\end{array}\right]\left[\begin{array}{cc}
c+s z_{3} & s z_{4} \\
0 & 1
\end{array}\right]=\left[\begin{array}{cc}
z_{1} & z_{2} \\
c z_{3}+s & z_{4}
\end{array}\right],
$$

equality of two matrices. Comparison of first entries in second rows results in

$$
s\left(z_{3}\right)^{2}=s .
$$

Since $g$ is not an identity transformation, $\left(z_{3}\right)^{2}=1$. If $z_{3}=1$, then comparison of first entries in the first rows results in $(c+s) z_{1}=z_{1}$, that is, $z_{1}=0$. Comparison of second entries in second rows results in $z_{4}=0$. Now, if $z_{3}=-1$ in (B.7), then, again, $z_{1}=z_{4}=0$.

Our next goal is to prove that each fixed point (of a given (B.1) - transfor-mation) is an extreme point of the conformal coefficient $h(z)$ : maximum is reached at each point of one circle whereas minimum is reached at each point of the other circle.

Scholium B.4. If $\left(u_{1}\right)^{2}+\left(u_{2}\right)^{2}<1$ at $z_{0}$, then $z_{0}$ is not a point of extremum for $h(z)$.

Proof. If $\left(u_{1}\right)^{2}+\left(u_{2}\right)^{2}<1$ at $z_{0}$, then $u_{1}, u_{2}$ can be chosen as two (of the total of four) free real variables at the vicinity of $z_{0}$. If $z_{0}$ is a point of extremum for $h(z)$, then each of the two partial derivatives of $h$ (w.r.t. $u_{1}$, and w.r.t. $u_{2}$ ) vanish at $z_{0}$. However, that would have resulted in vanishing of $h(z)$ at $z_{0}$.

Corollary B.5. If $z=\left(u_{-1}, u_{0}, u_{1}, u_{2}, u_{3}, u_{4}\right)$ is an extreme point for $h$, then $u_{3}=u_{4}=0$ (that is, $z_{1}=z_{4}=0$ ).

Corollary B.6. At the point of extremum for the conformal coefficient, either $u_{-1} u_{2}+u_{0} u_{1}=1$, or $u_{-1} u_{2}+u_{0} u_{1}=-1$ (that is, $z_{3}=-1$, or $z_{3}=1$ ).

Proof follows from the expression $h(z)=\left[c^{2}+s^{2}-2 s c\left(u_{-1} u_{2}+u_{0} u_{1}\right)\right]^{-1}$ which holds at every point $z$ where $u_{3}=u_{4}=0$.

Corollary B.7. An extreme value of $h(z)$ is reached at $z_{0}$ if and only if $z_{0}$ is a fixed point of (B.1). One can verify that the two extreme values are $e^{\tau}$ and $e^{-\tau}$ where $\tau$ is the (non-zero) value of the parameter in (B.1). 


\section{Appendix C: Evaluations of Integrals (2.8) for the Case of Appendix B Transformations in $E^{(2)}$}

We start with the form

$$
h=\left(c u_{-1}-s u_{2}\right)^{2}+\left(c u_{0}-s u_{1}\right)^{2}
$$

on the torus $T=S^{1} \times S^{3}$, see our Theorem B.1. Now, $T^{+}$is for the part of $T$ where $h \geq 1, T$ is for the part of $T$ where $h \leq 1$. Introduce

$$
\begin{aligned}
& I_{k}^{+}=\int_{T^{+}} h^{k}, \\
& I_{k}^{-}=\int_{T^{-}} h^{k} ;
\end{aligned}
$$

where in both cases we have in mind the volume form which has been introduced on $T$ in Section 2.

A word of caution: the function (C.1) is the inverse of the conformal coefficient (B.3). Nevertheless, the findings (which follow) of this Appendix C are relevant to the Appendix A content since $k$ in (C.2), (C.3) can be any integer.

The majority of these Appendix C findings are due to V. V. Ivanov (Sobolev Institute of Mathematics, Novosibirsk, Russia).

Parameterize $T$ as follows:

$$
\left.\begin{array}{l}
u_{-1}=\cos (\phi-\psi), u_{0}=\sin (\phi-\psi), u_{1}=\varrho \cos (\psi), u_{2}=\varrho \sin (\psi), \\
u_{3}=\sqrt{1-\varrho^{2}} \cos (\xi), \quad u_{4}=\sqrt{1-\varrho^{2}} \cos (\xi)
\end{array}\right\}
$$

In terms of these parameters, (C.1) becomes

$$
h=h(\varrho, \phi)=c^{2}-2 c s \varrho \sin (\phi)+s^{2} \varrho^{2} .
$$

The integrals (C.2), (C.3) are reduced as follows:

$$
I_{k}^{ \pm}=4 \pi^{2} J_{k}^{ \pm}
$$

where

$$
J_{k}^{ \pm}=\iint_{\Omega^{ \pm}} \varrho h^{k}(\varrho, \phi) \mathrm{d} \varrho \mathrm{d} \phi .
$$

Here we consider the rectangle $0 \leq \rho \leq 1,0 \leq \phi \leq 2 \pi$, and $\Omega^{+}=\{(\rho, \phi): h \geq 1\}, \Omega^{-}=\{(\rho, \phi): h \leq 1\}$. Notice that the integrals (C.6) and (C.7) are independent of the sign of $s=\sinh (\tau)$ which allows us to stay with $s>0$, only. The next step is to interpret $\rho, \phi$ as polar coordinates on the $x, y$ plane:

$$
x=\rho \cos (\phi), y=\rho \sin (\phi) \text {. }
$$

Our function (C.5) becomes

$$
h(x, y)=(s x)^{2}+(c-s y)^{2},
$$

whereas $\Omega^{+}, \Omega^{-}$are to be converted into $D^{+}, D^{-}$with their union being the unit disc $D$ centered at the origin $(0,0)$ of the $x, y$ plane. Finally, introduce coordinates $r, \alpha$ :

$$
x=r \sin (\alpha), y=c / s-r \cos (\alpha) .
$$

$r$ being the distance between $P=(0, c / s)$ and $Q=(x, y)$, whereas the angle $\alpha$, in radians, is an angle between vectors $\{0,-1\}$ and $P Q$. Expression (C.9) becomes

$$
h(r, \alpha)=s^{2} r^{2} .
$$

Introduce an (acute) angle $\omega$ which is determined by any of the relations

$$
\omega \cos (\omega)=1 / c, \quad \sin (\omega)=s / c, \quad \tan (\omega)=s .
$$

Omitting a few more (straightforward) technicalities, we obtain 


$$
J_{k}^{ \pm}=\left( \pm 2 / s^{2}\right) \int_{0}^{\omega} \int_{1}^{H_{ \pm}(\alpha)} r^{2 k+1} \mathrm{~d} r \mathrm{~d} \alpha .
$$

The upper limits $H_{+}(\alpha), H_{-}(\alpha)$ are as follows:

$$
H_{ \pm}(\alpha)=c \cos (\alpha) \pm \sqrt{c^{2} \cos ^{2} \alpha-1} .
$$

Let us conclude in terms of the following statements.

Theorem C.1. For $k$ not equal -1 , the integrals (C.2), (C.3) can be evaluated as follows:

$$
I_{k}^{ \pm}=\frac{ \pm 4 \pi^{2}}{(k+1) s^{2}} \int_{0}^{\omega}\left(H^{2 k+2}-1\right) \mathrm{d} \alpha .
$$

For $k=-1$

$$
I_{-1}^{ \pm}=\frac{ \pm 8 \pi^{2}}{s^{2}} \int_{0}^{\omega} \ln \left(H_{ \pm}(\alpha)\right) \mathrm{d} \alpha .
$$

Theorem C.2. For every integer $k$,

$$
I_{-k-2}^{+}=I_{k}^{-}, \quad I_{-k-2}^{-}=I_{k}^{+} .
$$

Theorem C.3. For a nonnegative $k$, each of the integrals (C.17) is a finite linear combination of integrals $A_{m}$, $B_{m}$ where

$$
\left.\begin{array}{l}
A_{m}=\int_{0}^{\omega} c^{2 m} \cos ^{2 m}(\alpha) \mathrm{d} \alpha, \\
B_{m}=\int_{0}^{\omega} c^{2 m-1} \cos ^{2 m-1}(\alpha) \sqrt{c^{2} \cos ^{2}(\alpha)-1} \mathrm{~d} \alpha .
\end{array}\right\}
$$

Remark C.4. Each of the integrals (C.20) is an elementary one and it can be expressed as a polynomial in $s$ and $\omega$.

Recall notations $a, b, c, d$ of Section 2 (see the line prior to Formula (2.9)) for the integrals which are of our utmost interest.

Theorem C.5. The integrals $a, b, c, d$, are as follows:

$$
\begin{gathered}
a=\left\{4 \pi^{2} /\left[3\left(s^{2}\right)\right]\right\}\left\{32 A_{3}-48 A_{2}+18 A_{1}-2 A_{0}+32 B_{3}-32 B_{2}+6 B_{1}\right\}, \\
b=4 \pi^{2}\left[\pi / 2+\omega+(s-\omega) /\left(s^{2}\right)\right], \\
c=\left\{4 \pi^{2} /\left[3\left(s^{2}\right)\right]\right\}\left\{32 A_{3}-48 A_{2}+18 A_{1}-2 A_{0}-32 B_{3}+32 B_{2}-6 B_{1}\right\}, \\
d=4 \pi^{2}\left[\pi / 2-\omega-(s-\omega) /\left(s^{2}\right)\right] .
\end{gathered}
$$

